\title{
Glycoprotein IIb/IIIa Antagonists in Acute Coronary Syndromes Undergoing PCI: A Long Way to Select Optimal Agent and Route
}

\author{
Paolo Emilio Puddu, ${ }^{1}$ Michele Schiariti, ${ }^{1}$ and Raffaele Bugiardini ${ }^{2}$ \\ ${ }^{1}$ Laboratrio di Biotechnologie Applicate alla Medicina Cardiovascolare, Dipartimento di Fisiopatologia Cardiocircolatoria, \\ Anestesiologia e Chirurgia, Istituto del Cuore "Attilio Reale", Sapienza Università di Roma, Rome 00161, Italy \\ ${ }^{2}$ Section of Cardiology, Department of Internal Medicine, University of Bologna, 40126 Bologna, Italy
}

Correspondence should be addressed to Paolo Emilio Puddu, paoloemilio.puddu@uniroma1.it

Received 24 March 2011; Accepted 9 May 2011

Academic Editors: J. L. Fleg and A. Hirata

Copyright (C) 2011 Paolo Emilio Puddu et al. This is an open access article distributed under the Creative Commons Attribution License, which permits unrestricted use, distribution, and reproduction in any medium, provided the original work is properly cited.

\begin{abstract}
Antiplatelet treatment in patients with an acute coronary syndrome (ACS), without or with ST segment elevation myocardial infarction (STEMI), forces to keep the balance between potential threats and optimal clinical advantages. Apart from clopidogrel, glycoprotein (GP) IIb/IIIa inhibitors (abciximab and 2 small molecules, tirofiban and eptifibatide) have come to the clinical scene. Recent evidence (2009-2011) is reviewed pointing to pharmacoeconometric considerations of concern in times of budget restrictions worldwide. In ACS, when clopidogrel plus aspirin are on, there might be no advantage to add small molecules. Whereas in STEMI patients treated by primary PCI, all 3 GP IIb/IIIa antagonists might be superimposable, when only ACS is present and PCI is elective, definite distinction among the 3 agents, both pharmacoeconomically and pharmacodynamically, might be invoked. There are still points open to debate. Among these the route (upstream versus downstream) is still a matter of uncertainties. Moreover, theoretically, there might be differences not only between abciximab and small molecules (mostly superimposable) but also between tirofiban and eptifibatide (the former being potentially more potent). Thus, a long way is needed before a prominent agent among GPIIb/IIIa inhibitors may be selected. The game is still open, a role will be played soon by new agents.
\end{abstract}

\section{Introduction}

Antiplatelet therapy has been shown to significantly reduce the risk of serious vascular events in high-risk patients, including those with a prior acute ischemic event and/or ST segment elevation myocardial infarction (STEMI). Longterm antiplatelet agents are key components of secondary prevention after acute coronary syndromes (ACS), especially when percutaneous coronary intervention (PCI) is looked for $[1,2]$. However, there might be a critical balance to monitor since any effective antiplatelet regimen may be closely related to increased risk for bleeding, often necessitating discontinuation of treatment and directly impinging upon a potentially worse long-term outcome [1]. It is therefore crucial for advised practitioners to keep the balance between potential threats and optimal clinical advantages in patients with ACS and/or STEMI when antiplatelet treatment is prescribed [2].
Glycoprotein (GP) IIb/IIIa antagonists including abciximab and the 2 small molecules tirofiban and eptifibatide have come to the clinical scene potently in the context of ACS and/or STEMI, but there might be a long way to go before the optimal agent is selected and the perfect strategy is defined to act effectively and at long term while keeping adverse consequences, mostly at short term, at the minimum. We address issues to be concretely derived from very recent evidence obtained in randomized clinical trials and registries while pointing to pharmacoeconometric considerations of concern in times of budget restrictions worldwide.

\section{Upstream Versus Downstream Administration of GP IIb/IIIa Inhibitors}

Recent contributions in patients with ACS have investigated whether upstream (before coronary angiography) 
administration of GP IIb/IIIa inhibitors is superior to their selective downstream administration during PCI. A meta-analysis of randomized trials from January 1990 to March 2010 included 7 mixed-up trials involving 19929 patients and compared upstream $(N=9981)$ to downstream $(N=9948)$ administration of GP IIb/IIIa inhibitors in highrisk patients with ACS undergoing early invasive strategy [2]. Upstream GP IIb/IIIa inhibitors did not decrease 30-day mortality (primary endpoint: $2.0 \%$ versus $2.0 \%, P=0.84$ ) or recurrence of myocardial infarction (secondary endpoint: $7.0 \%$ versus $7.6 \%, P=0.11$ ) but were associated with higher risk of major bleeding complications (1.8\% versus $1.3 \%$, $P=0.0002$ ). It seems therefore (Table 1 ) that a strategy of upstream GP IIb/IIIa inhibitors (overall considered) cannot be recommended [2] thus confirming, at least related to the downstream arm of the GP IIb/IIIa inhibitors, previous results obtained by the same authors who meta-analysed 16 randomized trials involving 10085 patients (5094 enrolled in the GP IIb/IIIa inhibitors group versus 4991 patients in the control group) [3]. GP IIb/IIIa inhibitors did not reduce 30day mortality (2.8 versus $2.9 \%, P=0.75$ ) or reinfarction (1.5 versus $1.9 \%, P=0.22$ ) but were associated with higher risk of major bleeding complications (4.1 versus $2.7 \%, P=$ $0.0004)$. Interestingly, a significant relationship was observed between patient's risk profile and benefits from adjunctive GP IIb/IIIa inhibitors in terms of death $(P=0.008)$ but not reinfarction $(P=0.25)$, pointing to the need to consider GP IIb/IIIa inhibitors especially among high-risk patients $[2,3]$.

\section{Clopidogrel on the Scene}

In patients with ACS undergoing PCI, it is essential to determine the optimal dose of antiplatelet therapy [1]. However, considerable interpatient variability in response to agents such as clopidogrel has been repeatedly reported. After $300 \mathrm{mg}$ clopidogrel loading dose followed by a $75 \mathrm{mg} /$ day maintenance dose in patients undergoing stenting, platelet function was assessed by measuring aggregation and the expression of activation-dependent receptors [5]. Using a cut point of $<10 \%$ absolute change in aggregation for resistance, the prevalence of resistance was about $50 \%$ to $60 \%$ at 2 hours, about $30 \%$ at 1 and 5 days, and $15 \%$ to $21 \% 30$ days after stenting [5]. Several trials were later performed to assess the optimal loading dose and timing of clopidogrel administration before PCI. The CREDO randomized trial was the first to establish the benefit of a longer time interval between loading dose administration and PCI [6]. However, adverse thrombotic events continued to occur despite the use of the standard $300 \mathrm{mg}$ loading dose of clopidogrel. The ALBION trial compared a $300 \mathrm{mg}$ loading dose of clopidogrel with 2 higher doses (600 and $900 \mathrm{mg}$ ) in 103 patients with non-STEMI [7]. The higher loading doses of clopidogrel were associated with more rapid and higher levels of inhibition of platelet aggregation, suggestive of a doseeffect relationship, greater reduction of platelet activation, no significant effect on variations of inflammatory markers, favorable trends on troponin release and ischemic events, and comparable safety profiles [7].
The impact of higher maintenance doses on platelet inhibition was evaluated in a double-blind study whereby 60 patients received long-term acetylsalicylic acid therapy after PCI and a $600 \mathrm{mg}$ loading dose of clopidogrel and were randomized to maintenance doses of 75 or $150 \mathrm{mg} /$ day of clopidogrel [8]. At 30 days following randomization, maximal $5 \mu \mathrm{mol} / \mathrm{L}$ ADP-induced platelet aggregation was significantly reduced, albeit with a large variability in platelet aggregation data, in patients treated with a $150 \mathrm{mg}$ maintenance dose of clopidogrel $(45.1 \% \pm 20.9 \%)$ compared with the conventional $(75 \mathrm{mg})$ dose $(65.3 \% \pm 12.1 \%, P<0.001)$ [8]. Similar results were obtained in diabetics with coronary artery disease [9]. There is no such information on different patients subgroups.

CURRENT OASIS 7 trial investigated whether doubling clopidogrel loading dose affected survival at short-term versus standard regimen [10]. There were 25087 unstable angina or acute myocardial infarction patients scheduled to undergo angiography within 72 hours of hospital arrival to a highdose regimen (600 mg loading dose of clopidogrel, followed by $150 \mathrm{mg} /$ day for 7 days) or the standard regimen (300 mg on the first day followed by $75 \mathrm{mg}$ /day later on). At 30 days, the combined rate of cardiovascular death, myocardial infarction, and stroke (primary endpoint) had occurred in $4.4 \%$ of patients on the standard-dose clopidogrel compared with $4.2 \%$ of patients on the high-dose (NS). Among the PCI patients of the study (around 9000 patients), the risk of stent thrombosis was reduced by $30 \%$ and the risk of myocardial infarction was reduced by $22 \%$ in the group that received the high dose compared with the group that received the standard dose, which was accompanied, as expected, by more major bleeding, yet no increase of fatal bleeds [10].

\section{Effects of GP IIb/IIIa Inhibitors or Different Agents on Top of Clopidogrel}

The safety of prehospital initiation of a high-dose tirofiban on top of aspirin, clopidogrel and heparin were assessed as a subanalysis of the On-TIME 2 trial where 1398 STEMI patients were enrolled and 1275 patients (91.2\%) had clinical follow up [11]. The independent association between bleeding and mortality (30-day and 1-year) was evaluated using Cox proportional Hazard models. Bleeding (major or minor) was observed in 47 patients $(3.7 \%)$, with only 13 patients (1\%) with major bleeding. The strongest independent determinants of bleeding were age (odds ratio (OR) $1.05,95 \%$ CI 1.01-1.08, $P=0.011)$, Killip class $>1$ at admission $(\mathrm{OR}=2.5,95 \% \mathrm{CI} 1.2-5.3, P=0.020)$, and intraaortic balloon pump (IABP) use (OR $=4.2,95 \%$ CI 1.6-11.1, $P=0.003)$. High-dose tirofiban was not an independent predictor of bleeding $(\mathrm{OR}=1.7,95 \%$ CI $0.9-3.2, P=$ $0.116)$. Bleeding was associated with an increased risk of 30 day mortality (HR 5.5, 95\% CI 1.6-7.8, $P<0.001$ ) and one-year mortality (HR 3.2, 95\% CI 1.4-7.2, $P=0.005$ ). These results of a single-centre investigation rather pointed to the usefulness of upstream use of high-dose tirofiban which was safe and associated with a low risk of bleeding [11]. Age, Killip class $>1$, and IABP use, but not highdose tirofiban, were independent determinants of bleeding in 
TABLE 1: Short-term (30-day) mortality plus nonfatal acute myocardial infarction with glycoprotein (GP) IIb/IIIa inhibitor's administration.

\begin{tabular}{|c|c|c|c|c|}
\hline Study name & Study type & Primary end-point & $\begin{array}{l}\text { Odds } \\
\text { ratio }\end{array}$ & $95 \% \mathrm{CI}$ \\
\hline Upstream versus downstream & & & 1.0 & $0.8-1.2$ \\
\hline ACUITY & RCT & 30-day death & 0.9 & $0.6-1.2$ \\
\hline CLOTILDA & RCT & 30-day death & 0.7 & $0.1-4.0$ \\
\hline EARLY ACS & RCT & 30-day death & 1.1 & $8.9-1.4$ \\
\hline ELISA & $\mathrm{RCT}$ & 30-day death & 0.6 & $0.1-2.6$ \\
\hline EARLY pilot & RCT & 30-day death & 5.2 & $0.2-109$ \\
\hline Liu et al. & $\mathrm{RCT}$ & 30-day death & 3.0 & $0.1-76$ \\
\hline ELISA 2 & RCT & 30-day death & 1.0 & $0.1-16$ \\
\hline Small molecules versus abciximab & & & 0.9 & $0.8-1.2$ \\
\hline Danzi et al. & RCT & 30-day ALL+NFami & 2.0 & $0.1-75$ \\
\hline Ernst et al. & RCT & 30-day ALL+NFami & 0.8 & $0.1-8.0$ \\
\hline EVA-AMI & $\mathrm{RCT}$ & 30-day ALL+NFami & 1.0 & $0.5-5.0$ \\
\hline MULTISTRATEGY & $\mathrm{RCT}$ & 30-day ALL+NFami & 0.6 & $0.2-2.0$ \\
\hline FATA & RCT & 30-day ALL+NFami & 2.0 & $0.6-8.0$ \\
\hline Raveedran et al. & $\mathrm{R}$ & 30-day ALL+NFami & 0.7 & $0.3-3.0$ \\
\hline Midei et al. & $\mathrm{R}$ & 30-day ALL+NFami & 2.0 & $0.6-7.0$ \\
\hline Lachance et al. & $\mathrm{R}$ & 30-day ALL+NFami & 1.5 & $0.4-7.0$ \\
\hline Gurm et al. & $\mathrm{R}$ & 30-day ALL+NFami & 0.8 & $0.6-1.5$ \\
\hline
\end{tabular}

Modified from $[2,4]$.

CI: confidence intervals; RCT: randomised clinical trial; R: registry; NFami: non fatal acute myocardial infarction. Note that odds ratios and CI were rounded off.

There was no significant advantage comparing administration modalities or type of GP IIb/IIIa antagonist (comparison confined to small molecules versus abciximab).

STEMI patients. Bleeding was independently associated with 30-day and 1-year mortality [11].

Lotrionte et al. recently presented a systematic review and meta-analysis of the optimal clopidogrel loading dose in patients scheduled for catheterization and/or PCI in the literature as of December 2006 [12]. Overall, a high loading dose had an efficacy, also among randomized trials alone, much higher than the standard loading dose: cardiac death or nonfatal myocardial infarction were prevented $(\mathrm{OR}=$ $0.54,95 \%$ CI $0.32-0.90, P=0.02$ ), without any statistically significant increase in major or minor bleedings $(P=0.55$ and $P=0.98$, resp.). Interestingly, metaregression disclosed a significant interaction between event rate and the benefits of high loading doses $(P=0.005)$, suggesting that the greater the underlying risk, the greater the favourable impact of a high loading dose. Therefore efficacy seemed dosedependent in spite of a dose-independent safety profile. Nevertheless, results over one month of these regimens remain unknown.

More recently, in patients with ACS with scheduled PCI, it was shown that with prasugrel, careful titration might be necessary to obtain a safety profile comparable to an efficacyequivalent dose of clopidogrel [13]. On the other hand, in STEMI patients undergoing primary PCI with bivalirudin or unfractionated heparin plus a GP IIb/IIIa inhibitor [14], a $600 \mathrm{mg}$ clopidogrel loading dose was an independent predictor of lower rate of 30-day major adverse cardiac events ( OR $=0.72,95 \%$ CI $0.53-0.98, P=0.04)$.

\section{Any Advantage from GP IIb/IIIa Antagonism in Diabetics or High-Risk STEMI Patients?}

Interestingly, a prespecified subanalysis of the randomised On-TIME trial $(n=984)$ and its open label run-in phase $(n=414)$, which investigated prehospital administration of high dose tirofiban in STEMI patients treated with primary PCI, included $220(16 \%)$ diabetic patients (known diabetes mellitus $(\mathrm{DM})$ or $\mathrm{Hba1C} \geq 6.2 \%)$ : there were 101 patients in the placebo group and 119 patients in the tirofiban group. In patients with DM, randomisation to tirofiban resulted in a lower residual ST deviation $(5.1 \pm 8.5 \mathrm{~mm}$ versus $6.2 \pm 5.6 \mathrm{~mm}, P=0.003)$, a reduced infarct size 
(CK 1694 $1925 \mathrm{U} / \mathrm{L}$ versus CK $2040 \pm 1829 \mathrm{U} / \mathrm{L}, P=0.02$ ), and a trend towards lower one-year mortality (4.6\% versus $11.6 \%, P=0.07$ ) [15]. Thus the beneficial effects of tirofiban were more pronounced in diabetic patients compared to patients without diabetes [15].

There is an undersized study in which 46 stable diabetic patients were enrolled while on double antiplatelet therapy and were either randomized to placebo $(N=22)$ or to high-dose bolus $(25 \mu \mathrm{g} / \mathrm{kg}$ per $3 \mathrm{~min})$ of tirofiban $(N=24)$ administered immediately before PCI followed by $8 \mathrm{~h}$ continuous infusion $(0.15 \mu \mathrm{g} / \mathrm{kg}$ per $\mathrm{min})$ [16]. Postprocedural myonecrosis was assessed prospectively by measurement of cardiac troponin $\mathrm{T}$ (cTnT) at 6 and $24 \mathrm{~h}$ after PCI. The primary endpoints were post-PCI coronary flow estimated by corrected thrombolysis in myocardial infarction frame count and post-PCI myocardial infarction. Post-PCI corrected thrombolysis in myocardial infarction frame count was similar in tirofiban and in placebo groups (10.2 \pm 3.6 versus $12.0 \pm 7.6, P=0.30$, resp.). The prevalence of raised cTnT levels was similar in the two groups (25 versus $30 \%$, $P=0.56$, resp.). Thus, a high-dose bolus of tirofiban in stable diabetic patients undergoing elective PCI was associated with a significant further inhibition of platelet aggregation which, however, did not translate into lower incidence of post-PCI distal embolization [16].

It has been pointed out, in STEMI patients, that the use of GP IIb/IIIa inhibitors continues to be challenged with the introduction of emerging antiplatelet therapy agents such as prasugrel and ticagrelor, which offer rapid onset with more reliable and more potent platelet inhibition compared with clopidogrel [17]. Additionally, both prasugrel and ticagrelor have been shown to reduce clinical events, including stent thrombosis rates, compared with clopidogrel [17]. An important question remains whether new antiplatelet therapies, which have rapid onsets and are easy to administer, will substitute the need for upstream GP IIb/IIIa inhibitor use prehospital or perhaps in the catheterization laboratory [17], although it is quite clear that interventional cardiologists are still comfortable with the use of GP IIb/IIIa inhibitors in combination with the new antiplatelet agents in high-risk STEMI patients.

\section{Abciximab Compared to Small Molecules}

Current guidelines recommend abciximab as an adjunctive pharmacologic agent to primary PCI for STEMI patients whereas small molecules are more commonly used in clinical practice [18]. It is of high interest therefore to compare abciximab versus small molecules, considering both safety and efficacy before primary PCI, which was done recently in a meta-analysis whereby the literature was scanned by formal searches of electronic databases from January 1990 to April 2009 [19]. A total of 4 randomized trials meeting the prespecified criteria were analyzed, involving 2040 patients. Rates of initial Thrombolysis in Myocardial Infarction Study (TIMI) 3 flow before procedure as well as complete ST resolution after primary PCI were not inferior in small molecules group compared with abciximab group $(\mathrm{OR}=$ $1.12, P=0.31 ; \mathrm{OR}=1.05, P=0.66$, resp.). There was no significant difference in the risk of 30-day $(\mathrm{OR}=0.83, P=$ 0.54 ) or 8 -month mortality $(\mathrm{OR}=0.78, P=0.43$ ) between small molecules and abciximab group. With regard to the safety endpoints, neither the major nor the minor bleeding complications in small molecules group differed significantly from those in abciximab group ( $\mathrm{OR}=1.32, P=0.43$; and $\mathrm{OR}=0.82, P=0.37$, resp.). Thus similar effectiveness was seen between small molecules and abciximab in the setting of primary PCI for STEMI, without an increase in bleeding complications [19].

\section{Which GP IIb/IIIa Agent for the Real Clinical World in STEMI Patients?}

A slightly different approach, closer to the real clinical world, was followed in a very recent and interesting meta-analysis whereby all randomized trials and registries (Table 1) were combined to demonstrate the noninferiority of tirofiban and eptifibatide compared to abciximab in patients with STEMI treated with primary PCI [4]. Overall, among 6 randomized trials and 4 registries, 4653 received small molecules and 2696 abciximab, and the rate of combined death and nonfatal reinfarction did not differ $(4.6 \%$ versus $4.5 \%$, OR $=0.99$, $95 \%$ CI $0.78-1.27, P=0.95)$ up to 30 days of followup, with an absolute difference of $0.1 \%$ (95\% CI - 1.06-0.8). Because the noninferiority limit was set at $+1.5 \%$ and because the upper point estimate $(0.8 \%)$ of the $95 \%$ CI did not cross the prespecified limit, the noninferiority of the small molecules was documented. In-hospital major bleeding was also similar (8.8\% versus $6.1 \%, \mathrm{OR}=0.92,95 \%$ CI $0.75-1.13, P=$ $0.43)$. No significant differences were seen by sensitivity analysis when comparing randomized trials to registries and tirofiban or eptifibatide to abciximab. These results documented noninferiority of small molecules compared to abciximab [4] and, therefore, support their alternative use as adjunctive therapy during primary PCI for patients with STEMI.

\section{Which GP IIb/IIIa Agent in ACS (without or with STEMI) Treated by PCI?}

Randomized trials comparing tirofiban versus placebo or any active control were actively searched, and OR were computed from individual studies and pooled with random-effect methods [20]. Thirty-one studies were identified involving 20006 patients (12874 comparing tirofiban versus heparin plus placebo or bivalirudin alone, and 7132 versus abciximab). When compared with placebo, tirofiban was associated at 30 days with a significant reduction in mortality $(\mathrm{OR}=0.68$ (95\% CI: 0.54-0.86); $P=0.001)$ and death or myocardial infarction $(\mathrm{OR}=0.69(0.58-0.81) ; P<0.001)$. The treatment benefit persisted at followup but came at an increased risk of minor bleedings ( $\mathrm{OR}=1.42(1.13-1.79)$, $P=0.002)$ or thrombocytopenia. When compared with abciximab, mortality at 30 days did not differ $(\mathrm{OR}=0.90$ (0.53-1.54); $P=0.70)$, but, in the overall group tirofiban trended to increase the composite of death or myocardial infarction $(\mathrm{OR}=1.18(0.96-1.45) ; P=0.11)$. No such trend 
persisted at medium-term followup or when appraising studies testing tirofiban at $25 \mu \mathrm{g} / \mathrm{kg}$ bolus regimen. Thus, tirofiban administration reduced mortality, the composite of death, or myocardial infarction and increased minor bleedings when compared with placebo. An early ischemic hazard disfavouring tirofiban was noted when compared with abciximab in studies based on 10 but not $25 \mu \mathrm{g} / \mathrm{kg}$ tirofiban bolus regimen [20].

Comparisons between abciximab and eptifibatide were undertaken quite recently either assessing randomly assigned differences in a largely undersized single-centre investigation [21] or investigating noninferiority in a large nationwide registry of STEMI patients treated by primary PCI [22]. A total of 427 patients with ST-segment elevation myocardial infarctions $<12 \mathrm{~h}$ and planned primary PCI were randomized to double-bolus eptifibatide $(n=226)$ followed by a $24-\mathrm{h}$ infusion or single-bolus abciximab $(n=201)$ followed by a $12-\mathrm{h}$ infusion. In this noninferiority trial, the primary endpoint was the incidence of complete ( $>$ or $=70 \%$ ) ST-segment resolution (STR) 60 min after PCI, a measure of myocardial reperfusion. The assumption was a $60 \%$ complete STR rate in the abciximab group. The noninferiority margin was set to $15 \%$. All-cause mortality $6.2 \%$ versus $4.5 \%(P=$ $0.50)$; reinfarction $0.4 \%$ versus $3.5 \%(P=0.03)$; target vessel revascularization $4.4 \%$ versus $6.5 \%(P=0.40)$; the combined endpoint of death, nonfatal reinfarction, and target vessel revascularization $10.6 \%$ versus $10.9 \%(P=$ $0.90)$; stroke $0.5 \%$ versus $0.5 \%(P=1.00)$ after 6 months; TIMI major bleeding complications $4.0 \%$ versus $2.0 \%(P=$ 0.20 ) after 30 days were observed after eptifibatide and abciximab, respectively. Thus, eptifibatide as an adjunct to primary PCI was equally as effective as abciximab with respect to STR [21].

On the other hand, all $(n=11479)$ STEMI patients in Sweden who underwent primary PCI and received either eptifibatide or abciximab from 2004 to 2007 were studied. The primary end point was death or myocardial infarction during 1-year followup, with adjustment for baseline differences with a multivariate logistic regression analysis including propensity score. The prespecified noninferiority margin was set to 1.29 . The combined endpoint occurred in 353 of 2355 patients $(15.0 \%)$ treated with eptifibatide and in 1432 of 9124 patients $(15.7 \%)$ treated with abciximab. The unadjusted OR for eptifibatide versus abciximab was 0.95 (95\% CI: 0.84 to 1.08 ). Multivariate adjustment confirmed noninferiority, with an OR of 0.94 (95\% CI: 0.82 to 1.09). The adjusted secondary endpoints of death and myocardial infarction separately also showed noninferiority, with OR of 0.99 (95\% CI: 0.82 to 1.19 ) and 0.88 (95\% CI: 0.73 to 1.05$)$, respectively. Thus, eptifibatide was noninferior to abciximab in patients with STEMI undergoing primary PCI with respect to death or myocardial infarction during 1 year [22].

The Sant'ANna TIrofiban Safety study (SANTISS) was an open-label investigator-initiated single-centre registry launched to assess the combination of bleeding and access site in-hospital complications (primary endpoint) in patients undergoing PCI by femoral approach only [23]. Patients who were on oral single antiaggregating drug (AAD) and received, just prior to $\mathrm{PCI}$, high-dose tirofiban and a second oral antiplatelet agent (triple AAD: group $1, N=970$ ) are compared with those who were already on an oral double $\mathrm{AAD}$ regimen and did not receive tirofiban (double AAD: group $2, N=608$ ). Group 2 patients were slightly older, presented less frequently with unstable angina, and had chronic renal failure more frequently. They were more than twice as frequently on rescue PCI, being more than three-fold less frequently on primary PCI ( $P$ between 0.001 and 0.01 ). Overall, there were 87 in-hospital (average 4.7 days of stay) complications: 51 (5.3\%) in group 1 and 36 (5.9\%) in group 2 (not significant). Blood transfusions were needed in 34 patients: $21(2.2 \%)$ in group 1 and $13(2.1 \%)$ in group 2 (not significant). Of the 16 hospital deaths, eight ( $0.8 \%)$ were seen in group 1 and eight (1.3\%) in group 2 (not significant). Multivariate prediction showed a high predictive accuracy (areas under the curve $>0.700$ ) of female sex, rescue PCI, and chronic renal failure to index complications, with highly significant odds ratios. The presence of high-dose tirofiban did not increase complication risk. Thus, in ACS patients from the real world, high-dose tirofiban was well tolerated by patients on elective, primary, or rescue PCI, and the inhospital complication rate, including major bleeding, was low [23].

The ability of tirofiban or eptifibatide to reduce the incidence of composite ischemic events within one year in patients undergoing PCI in the real clinical world was further investigated [24]. Consecutive patients on oral double AAD (with clopidogrel and aspirin) who underwent PCI $(n=207)$ and patients who were on single $\mathrm{AAD}$ and received a second $\mathrm{AAD}$, just prior to $\mathrm{PCI}$, and either high-dose tirofiban or double-bolus eptifibatide (double AAD plus small molecules group, $n=666$ ), were compared. The primary endpoint (incidence of composite ischemic events within one year) included death, acute myocardial infarction, unstable angina, stent thrombosis or repeat PCI or coronary bypass surgery (related to the target vessel PCI failure) and was modelled by Cox's regression. There were 89 composite ischemic events: $24(11.6 \%)$ in double AAD alone and $65(9.8 \%)$ in double AAD plus small molecules groups (log-rank test: $P=0.36)$. Incidences by type of ischemic events were similar between the 2 groups. Based on 21 potential covariates fitted simultaneously, adjusted hazard ratios (HR and 95\% CI) showed that age (HR 1.03, 1.01-1.06, $P=0.01)$, diabetes (HR 1.68, 1.01-2.79, $P=0.05$ ), and IABP (HR 5.12, 2.36$11.10, P=0.0001)$ were significant risk factors, whereas thrombolysis by tenecteplase (HR 0.35, 0.13-0.98, $P=0.05$ ) and having had hypertension or antihypertensive treatment (HR 0.58, 0.36-0.93, $P=0.03$ ) were significant protectors for events. Whether small molecules were present provided a nonsignificant additional benefit as compared to double AAD alone (HR 0.83, 0.51-1.36, $P=0.46$ ). Pre-PCI CK$\mathrm{MB}$ was not useful to predict events (HR 1.01, 0.99-1.01, $P=0.17)$. Thus, in clinical world patients undergoing PCI (rescue plus primary $<13 \%$ ) while on double AAD, based on clopidogrel plus aspirin, small molecules (tirofiban or eptifibatide) provided no additive long-term protection against the occurrence of composite ischemic events whereas thrombolysis by tenecteplase did [24]. 


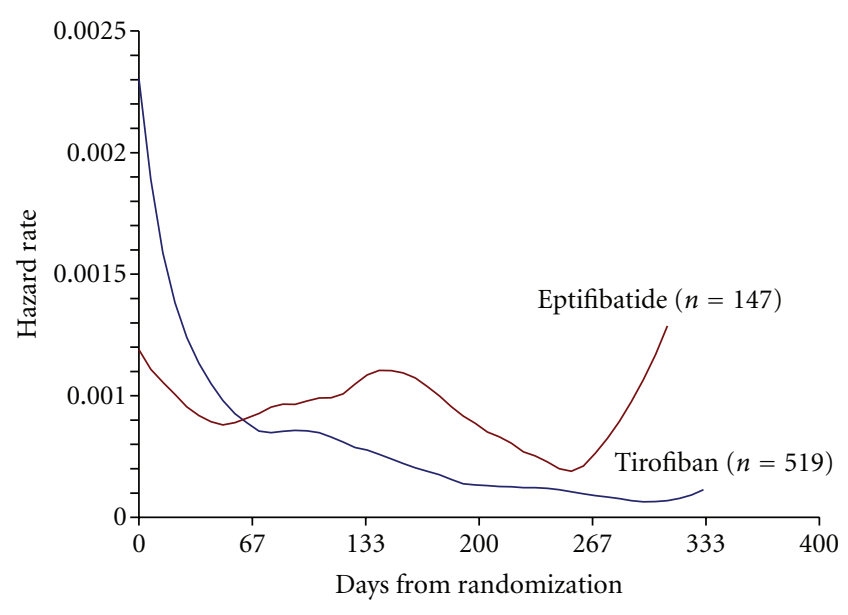

Figure 1: Comparison between eptifibatide and tirofiban patients shows a continuous decreasing hazard rate, from immediately after percutaneous coronary intervention to one year afterwards, in the tirofiban group. Eptifibatide patients have two late phases of hazard rate increase. This experience is from the study by Schiariti et al. [32].

\section{Economic Evaluation}

There might be elements to justify the decline of GP IIb/IIIa inhibitors use during PCI in recent years, mostly based on the incremental use of clopidogrel (also at high dosage, as discussed above) and aspirin but also on the appearance of new potent and relatively safer antiplatelet agents $[1,3$, 25]. However, 1 of the 3 approved GP IIb/IIIa inhibitors was used in about $39 \%$ of PCI procedures during 2008, as reported in June 2010, and the market is still a "big business" with more than $\$ 475$ million in the US alone in 2008 and definitely much more worldwide [26]. The average wholesale price of abciximab (ReoPro: Eli Lilly and Company, Indianapolis, Ind.) is $\$ 1836,9$ (for a 12 -h infusion) and those of eptifibatide (Integrilin: Schering-Plough, Kenilworth, NJ) and tirofiban (Aggrastat: Medicure, Winnipeg, Canada) are, respectively, $\$ 1121,81$ and 711,72 (for 18-h infusion) when calculated for $80 \mathrm{Kg}$ patients with normal renal function [26]. If 2 treatments are similar in efficacy and safety, it is highly probable that price, in this era of health care budgetary restrictions, is the most important consideration to lead the choice, which justifies a fierce war among involved companies and salespeople who become more friendly and/or attractive to be effective in persuasion [26]. The situation will probably not change in the years to come since in order to show noninferiority of 2 agents (if any small but clinically significant margin of noninferiority were chosen), a trial comparing 2 GP IIb/IIIa agents should enrol more than 10000 patients [26] which is demanding not only for multicentre cooperation but also for a tremendous investment so that it is very unlikely that one will ever be started.

\section{Pharmacodynamics}

GP IIb/IIIa inhibitors were developed so rapidly that phase 1 and phase 2 studies were at most deficient, and as a con- sequence the "wrong" or suboptimal doses for all of the 3 compounds were rushed into clinical practice [27], which resulted in inconclusive results of randomized trials also when they were appropriately sized as was the case with the TARGET (Do Tirofiban and ReoPro Give Similar efficacy Trial) [28]. Apart from the problem of dosing, which is now largely resolved for all 3 compounds, there are important differences in the pharmacodynamics of GP IIb/IIIa inhibitors [27]. These make abciximab, which is a very large molecule, so effectively tied to platelets that very little unbound compound remains circulating in the bloodstream [27], at least theoretically making the small molecules (particularly so tirofiban) comparatively more liable to respond to new platelets, since there are as many as 200 molecules of circulating drug for every molecule that binds to a receptor [27]. A situation that may prevent fibrinogen molecules to bind GP IIb/IIIa receptors and crosslink with other platelets, thus preventing clot formation [27].

\section{Are There Differences between Small Molecules?}

Although there might be an important difference between tirofiban and eptifibatide, the latter binding less effectively to GP IIb/IIIa receptors with molecules of eptifibatide releasing and rebinding the platelet receptor, which may increase the risk of clot formation and particularly so when the dose is under the optimal range [27], it has long been asked whether there might be clinical differences to justify a selection between small molecules [29-31]. However, it is highly probable that the war envisaged [26] in the market place between and among companies involved in the GP IIb/IIIa inhibitor world will prevent any direct comparison to be undertaken between tirofiban and eptifibatide if until now no such studies were sustained, a situation which may continue also in the future. Accordingly, we summarize the results of the only recent, yet relatively undersized, study [32] in patients undergoing PCI. In that investigation, tirofiban versus eptifibatide were compared on top of standard AAD to see whether ischemic events were prevented within 1 year. Patients undergoing PCI who were on oral single AAD were block randomized to receive, immediately preintervention, high-dose tirofiban $(N=519)$ or double-bolus eptifibatide $(N=147)$ and a second oral antiplatelet agent. The incidence of composite ischemic events within 1 year, including death, acute myocardial infarction, angina, stent thrombosis or repeat PCI or coronary bypass surgery (primary end-point), was modelled by forced Cox's regression. There were overall 65 composite ischemic events: $47(9.1 \%)$ in the tirofiban group and $18(12.2 \%)$ in the eptifibatide group (univariate log-rank test: $P=0.22$ ). Figure 1 shows that there was a continuous decreasing hazard rate, from immediately after PCI to 1 year afterwards in the tirofiban group, whereas eptifibatide patients had two late phases of hazard rate increase.

Based on 21 potential covariates fitted simultaneously [32], multivariable adjusted hazard ratios showed that age (HR 1.03, 95\% CI 1.01-1.07, $P=0.01$ ), chronic renal failure 
(HR 3.21, 95\% CI 1.02-10.10, $P=0.05)$, pre-PCI values of creatine kinase-myocardial band (CK-MB) (HR 1.002, 95\% CI $1.0002-1.0054, P=0.04$ ), IABP (HR ratio $5.88,95 \% \mathrm{CI}$ $12.33-14.85, P=0.0002$ ), and the presence of eptifibatide (HR 1.85, 95\% CI 1.04-3.29, $P=0.04$ ) were significant risk factors whereas thrombolysis by tenecteplase (hazard ratio $0.19,95 \%$ CI $0.05-0.69, P=0.01$ ) was a significant protector. Interestingly, eptifibatide versus tirofiban efficacy was explained based on pre-PCI values of CK-MB [32]. It might be of interest to compare, by meta-analysis, the results of randomized trials and of registries in ACS patients either receiving tirofiban or eptifibatide, to see whether short- or long-term effects are, respectively, noninferior. This may lead objective support for selection.

\section{A Long Way to Go?}

The most recently updated STEMI and PCI guidelines were in favour of indicating that the $3 \mathrm{GP}$ IIb/IIIa inhibitors have similar effectiveness in the setting of primary PCI [33], although individual opinions call attention to the fact that the evidence may not be considered as level 1 [26]. In ACS, it might appear that when clopidogrel plus aspirin are on, there might be no advantage to add small molecules [24]. In addition, whereas in STEMI patients treated by primary PCI all $3 \mathrm{GP}$ IIb/IIIa antagonists might be superimposable [33], when only ACS is present and PCI is elective, other factors may be involved and definite distinction among the 3 agents, both pharmacoeconomically and pharmacodynamically, as discussed earlier, might be invoked [26, 27, 29-31]. Interventional cardiologists may thus select a given GP IIb/IIIa antagonist based on a balanced comparison of the trials (and sometimes of the registries) more recently published. Others will accept a promising new but less completely investigated agent. There will be those who will select the cheapest agent, and there might be some, hopefully few, who will make selection based more on salespeople presentation and attractiveness than on personal meditation of bulk evidence or independently presented reviews.

No doubt, the dosages of any of the selected GP IIb/ IIIa antagonists need be in the full therapeutic range in order to prevent not only potential lower efficacy but also complications [26]. There are still points open to debate. Among these the route (upstream versus downstream) is still a matter of uncertainties $[2,11,17]$. Moreover, it is important to stress that, theoretically [27], there might be differences not only between abciximab and small molecules (mostly superimposable) but also between tirofiban and eptifibatide (the former being potentially more potent). Although several studies are under way to look for new indications and routes [34-36], it is probable that direct comparison in appropriately sized randomized trials between or among agents will not be ready in the future. We will rely for selection on meta-analyses and on registries, which is a suboptimal way to reach proper conclusions. Finally, it is for further research to assess comparatively the higher doses of clopidogrel (600 and $900 \mathrm{mg}$ investigated in the ALBION trial [7]) versus GPIIb/IIIa inhibitors both at short and long terms. In conclusion, we may state that a long way is needed before we can select a prominent agent among GPIIb/IIIa inhibitors. The game is still open, and new agents will play a role in the near future.

\section{References}

[1] M. Valgimigli and M. Minarelli, "Antiplatelet and antithrombotic treatment after primary percutaneous coronary intervention: balancing safety and efficacy," American Heart Journal, vol. 160, no. 6, pp. S36-S41, 2010.

[2] G. De Luca, E. P. Navarese, E. Cassetti, M. Verdoia, and H. Suryapranata, "Meta-analysis of randomized trials of glycoprotein IIb/IIIa inhibitors in high-risk acute coronary syndromes patients undergoing invasive strategy," American Journal of Cardiology, vol. 107, no. 2, pp. 198-203, 2011.

[3] G. De Luca, E. Navarese, and P. Marino, "Risk profile and benefits from Gp IIb/IIIainhibitors among patients with STsegment elevation myocardial infarction treated with primary angioplasty: a meta-regression analysis of randomized trials," European Heart Journal, vol. 30, no. 22, pp. 2705-2713, 2009.

[4] F. Ottani, L. La Vecchia, M. De Vita, O. Catapano, F. Tarantino, and M. Galvani, "Comparison by meta-analysis of eptifibatide and tirofiban to abciximab in patients with st-elevation myocardial infarction treated with primary percutaneous coronary intervention," American Journal of Cardiology, vol. 106, no. 2, pp. 167-174, 2010.

[5] V. L. Serebruany, S. R. Steinhubl, P. B. Berger, A. I. Malinin, D. L. Bhatt, and E. J. Topol, "Variability in platelet responsiveness to clopidogrel among 544 individuals," Journal of the American College of Cardiology, vol. 45, no. 2, pp. 246-251, 2005.

[6] S. R. Steinhubl, P. B. Berger, J. T. Mann III et al., "Early and sustained dual oral antiplatelet therapy following percutaneous coronary intervention: a randomized controlled trial," JAMA, vol. 288, no. 19, pp. 2411-2420, 2002.

[7] G. Montalescot, G. Sideris, C. Meuleman et al., "A randomized comparison of high clopidogrel loading doses in patients with non-ST-segment elevation acute coronary syndromes: the ALBION (Assessment of the Best Loading Dose of Clopidogrel to Blunt Platelet Activation, Inflammation and Ongoing Necrosis) trial," Journal of the American College of Cardiology, vol. 48, no. 5, pp. 931-938, 2006.

[8] N. von Beckerath, A. Kastrati, A. Wieczorek et al., "A doubleblind, randomized study on platelet aggregation in patients treated with a daily dose of 150 or $75 \mathrm{mg}$ of clopidogrel for 30 days," European Heart Journal, vol. 28, no. 15, pp. 1814-1819, 2007.

[9] D. J. Angiolillo, S. B. Shoemaker, B. Desai et al., "Randomized comparison of a high clopidogrel maintenance dose in patients with diabetes mellitus and coronary artery disease: results of the optimizing antiplatelet therapy in diabetes mellitus (OPTIMUS) study," Circulation, vol. 115, no. 6, pp. 708-716, 2007.

[10] S. F. Mehta, F. Van de Werf et al., "A randomized comparison of a clopidogrel high loading and maintenance dose regimen versus standard dose and high versus low dose aspirin in 25,000 patients with acute coronary syndromes: results of the CURRENT OASIS 7 Trial," Tech. Rep., European Society of Cardiology, Barcelona, 2009, http://www.escardio.org/congresses/esc-2009/congress-reports/Pages/706003-706004-mehta-vandewerf.aspx\#discussant.

[11] R. S. Hermanides, J. P. Ottervanger, and J. P. Dambrink, "Risk of bleeding after prehospital administration of high dose 
tirofiban for ST elevation myocardial infarction," International Journal of Cardiology. In press.

[12] M. Lotrionte, G. G. L. Biondi-Zoccai, P. Agostoni et al., "Metaanalysis appraising high clopidogrel loading in patients undergoing percutaneous coronary intervention," American Journal of Cardiology, vol. 100, no. 8, pp. 1199-1206, 2007.

[13] S. D. Wiviott, D. Trenk, A. L. Frelinger et al., "Prasugrel compared with high loading- and maintenance-dose clopidogrel in patients with planned percutaneous coronary intervention: the prasugrel in comparison to clopidogrel for inhibition of platelet activation and aggregation-thrombolysis in myocardial infarction 44 trial," Circulation, vol. 116, no. 25, pp. 29232932, 2007.

[14] G. Dangas, R. Mehran, G. Guagliumi et al., "Role of clopidogrel loading dose in patients with ST-segment elevation myocardial infarction undergoing primary angioplasty: results from the HORIZONS-AMI (harmonizing outcomes with revascularization and stents in acute myocardial infarction) trial," Journal of the American College of Cardiology, vol. 54, no. 15, pp. 1438-1446, 2009.

[15] J. R. Timmer, J. Ten Berg, A. A. Heestermans et al., "Prehospital administration of tirofiban in diabetic patients with ST-elevation myocardial infarction undergoing primary angioplasty: a sub-analysis of the On-Time 2 trial," EuroIntervention, vol. 6, pp. 336-342, 2010.

[16] G. P. Talarico, M. Brancati, F. Burzotta et al., "Glycoprotein IIB/IIIA inhibitor to reduce postpercutaneous coronary intervention myonecrosis and improve coronary flow in diabetics: the "OPTIMIZE-IT" pilot randomized study," Journal of Cardiovascular Medicine, vol. 10, no. 3, pp. 245-251, 2009.

[17] R. Waksman, "IIb or Not IIb: is there a place for pre-hospital tirofiban in ST-segment elevation myocardial infarction patients?" Journal of the American College of Cardiology, vol. 55, no. 22, pp. 2456-2458, 2010.

[18] S. C. Smith Jr., T. E. Feldman, J. W. Hirshfeld Jr. et al., "ACC/ AHA/SCAI 2005 guideline update for percutaneous coronary intervention: a report of the American College of Cardiology/American Heart association Task Force on Practice Guidelines (ACC/AHA/SCAI writing Committee to update the 2001 Guidelines for percutaneous coronary intervention)," Journal of the American College of Cardiology, vol. 47, no. 1, pp. e1-e121, 2006.

[19] L. Dong, F. Zhang, and X. Shu, "Early administration of smallmolecule glycoprotein IIb/IIIa inhibitors before primary percutaneous coronary intervention for st-elevation myocardial infarction: insights from randomized clinical trials," Journal of Cardiovascular Pharmacology and Therapeutics, vol. 15, no. 2, pp. 135-144, 2010.

[20] M. Valgimigli, G. Biondi-Zoccai, M. Tebaldi et al., "Tirofiban as adjunctive therapy for acute coronary syndromes and percutaneous coronary intervention: a meta-analysis of randomized trials," European Heart Journal, vol. 31, no. 1, pp. 3549, 2010.

[21] U. Zeymer, A. Margenet, M. Haude et al., "Randomized comparison of eptifibatide versus abciximab in primary percutaneous coronary intervention in patients with acute STsegment elevation myocardial infarction: results of the EVAAMI trial," Journal of the American College of Cardiology, vol. 56, no. 6, pp. 463-469, 2010.

[22] A. Akerblom, S. K. James, M. Koutouzis et al., "Eptifibatide is noninferior to abciximab in primary percutaneous coronary intervention: results from the SCAAR (Swedish Coronary Angiography and Angioplasty Registry)," Journal of the American College of Cardiology, vol. 56, no. 6, pp. 470-475, 2010.

[23] M. Schiariti, A. Saladini, B. Missiroli et al., "Safety of downstream high-dose tirofiban bolus among 1578 patients undergoing percutaneous coronary intervention: the Sant'ANna TIrofiban safety study," Journal of Cardiovascular Medicine, vol. 11, no. 4, pp. 250-259, 2010.

[24] M. Schiariti, A. Saladini, F. Papalia et al., "GPIIb/IIIa receptor antagonism using small molecules provides no additive longterm protection after percutaneous coronary intervention as compared to clopidogrel plus aspirin," Open Cardiovascular Medicine Journal, vol. 4, pp. 151-156, 2010.

[25] L. De Luca, G. Casella, M. Lettino et al., "Clinical implications and management of bleeding events in patients with acute coronary syndromes," Journal of Cardiovascular Medicine, vol. 10, no. 9, pp. 677-686, 2009.

[26] P. B. Berger, "The glycoprotein IIb/IIIa inhibitor wars," Journal of the American College of Cardiology, vol. 56, no. 6, pp. 476478, 2010.

[27] D. J. Schneider and A. Aggarwal, "Development of glycoprotein IIb/IIIaantagonists: translation from pharmacodynamic effect to clinical benefit," Expert Review of Cardiovascular Therapy, vol. 2, no. 6, pp. 903-913, 2004.

[28] E. J. Topol, D. J. Moliterno, H. C. Herrmann et al., "Comparison of two glycoproteins IIb/IIIa inhibitors, tirofiban and abciximab, for the prevention of ischemic events with percutaneous coronary revascularization," The New England Journal of Medicine, vol. 344, no. 25, pp. 1888-1894, 2001.

[29] C. Le Pen and H. Lilliu, "GPIIb/IIIa antagonist in percutaneous coronary intervention: how should economic criteria be factored in?" Pharmacy World and Science, vol. 27, no. 2, pp. 83-91, 2005.

[30] S. J. Brener, "The benefits of platelet glycoprotein IIb/IIIa receptor inhibition during primary percutaneous coronary intervention for ST-segment elevation myocardial infarction: drug-specific or class effect?" Journal of the American College of Cardiology, vol. 53, no. 18, pp. 1674-1676, 2009.

[31] F. Zijlstra, "The benefits and risks of Abciximab, eptifibatide, or tirofiban during PCI: class effects or do they differ?" Catheterization and Cardiovascular Interventions, vol. 73, no. 2, pp. 222-223, 2009.

[32] M. Schiariti, A. Saladini, D. Cuturello, B. Missiroli, and P. E. Puddu, "Long-term efficacy of high-dose tirofiban versus double-bolus eptifibatide in patients undergoing percutaneous coronary intervention," Journal of Cardiovascular Medicine, vol. 12, pp. 29-36, 2011.

[33] F. G. Kushner, M. Hand, S. C. Smith Jr et al., "Focused updates: ACC/AHA guidelines for the management of patients with STelevation myocardial infarction (updating the 2004 guideline and 2007 focused update) and ACC/AHA/SCAI guidelines on percutaneous coronary intervention (updating the 2005 guideline and 2007 focused update) a report of the American College of Cardiology Foundation/American Heart Association Task Force on Practice Guidelines," Journal of the American College of Cardiology, vol. 54, pp. 2205-2241, 2009.

[34] G. G. Biondi-Zoccai, M. Valgimigli, I. Sheiban et al., "A randomized trial comparing eptifibatide versus placebo in patients with diffuse coronary artery disease undergoing drugeluting stent implantation: design of the INtegrilin plus STenting to Avoid myocardial Necrosis Trial," Journal of Cardiovascular Medicine, vol. 9, no. 9, pp. 957-962, 2008. 
[35] C. D’Andrea, G. Esposito, F. Piscione, and M. Chiariello, "Tirofiban administration and percutaneous coronary intervention with stenting of saphenous vein graft thrombosis," Journal of Cardiovascular Medicine, vol. 10, no. 11, pp. 875$878,2009$.

[36] G. Sardella, G. M. Sangiorgi, M. Mancone et al., "A multi center randomized study to evaluate intracoronary abciximab with the ClearWay catheter to improve outcomes with Lysis (IC ClearLy): trial study design and rationale," Journal of Cardiovascular Medicine, vol. 11, pp. 529-535, 2010. 


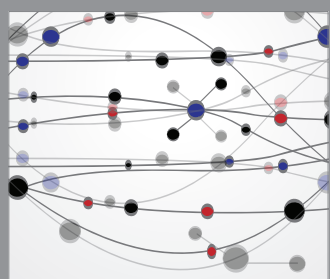

The Scientific World Journal
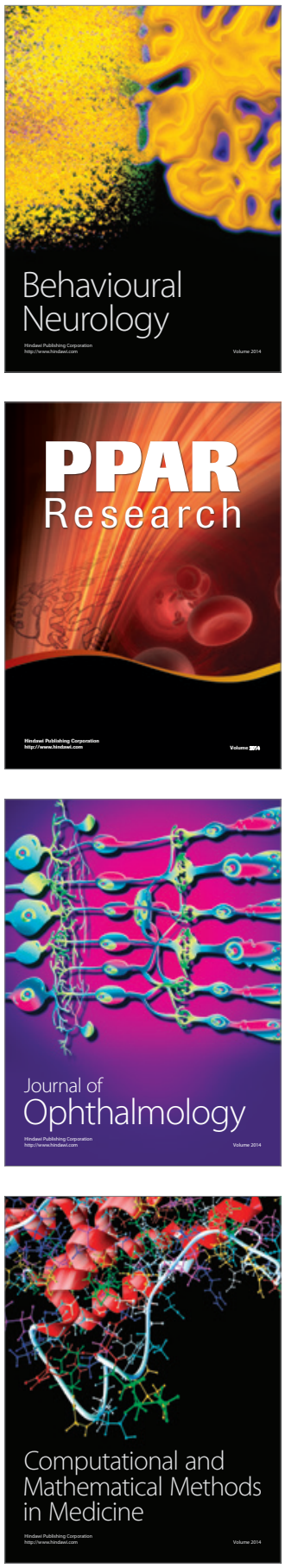

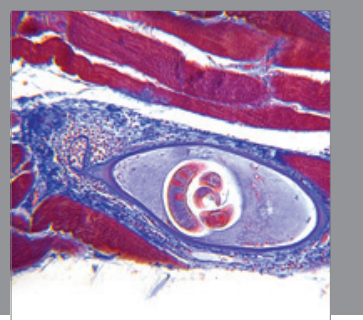

Gastroenterology

Research and Practice
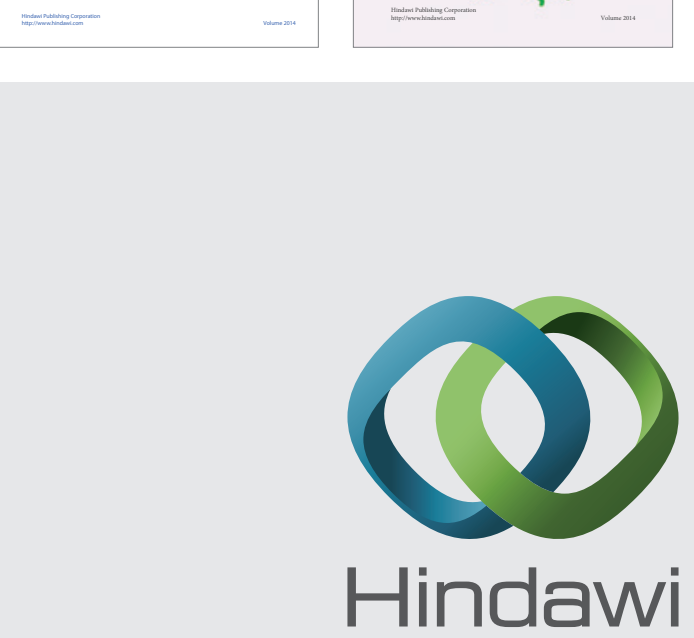

Submit your manuscripts at

http://www.hindawi.com
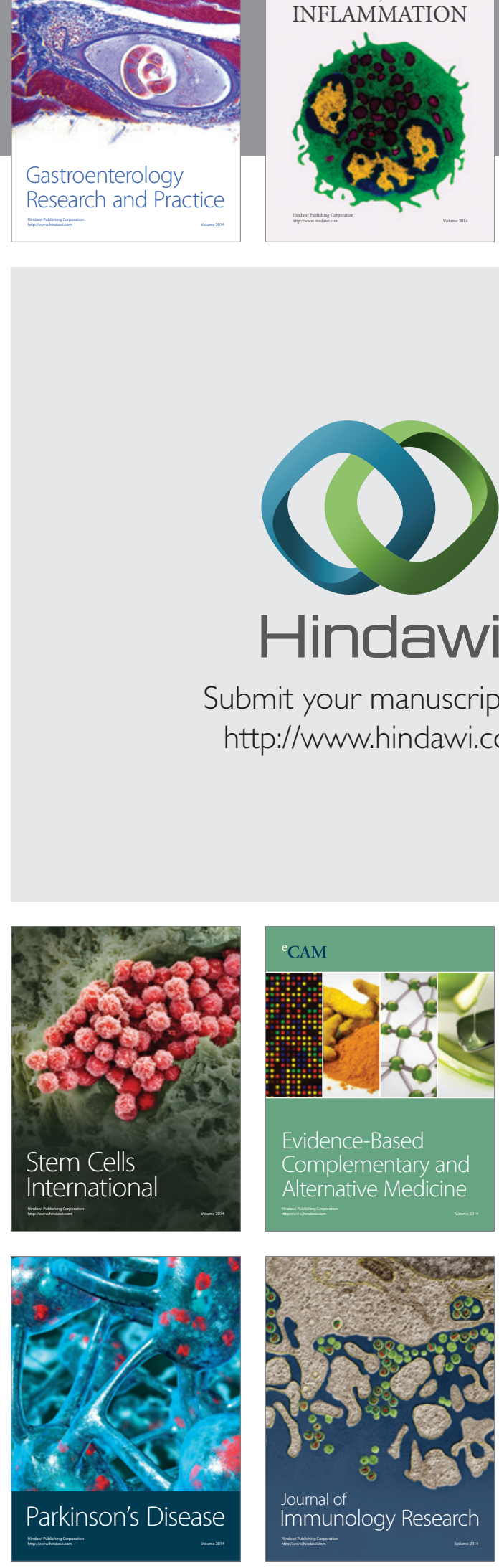

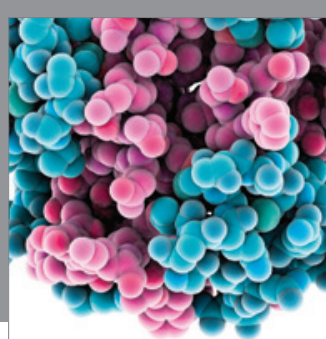

Diabetes Research
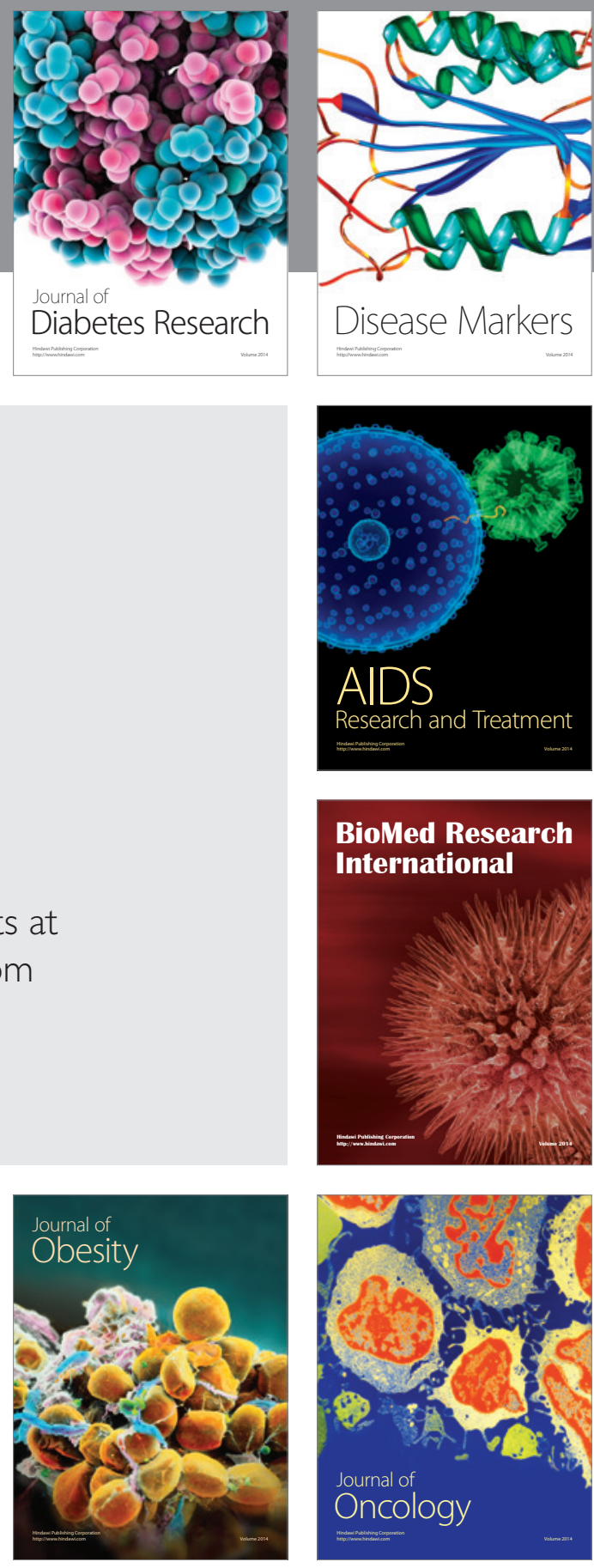

Disease Markers

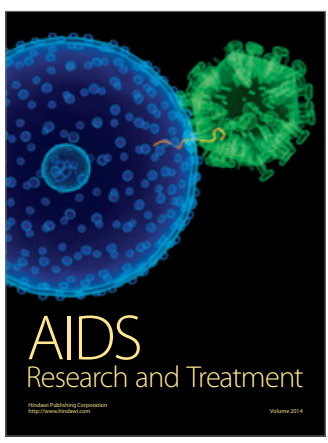

BioMed Research

International
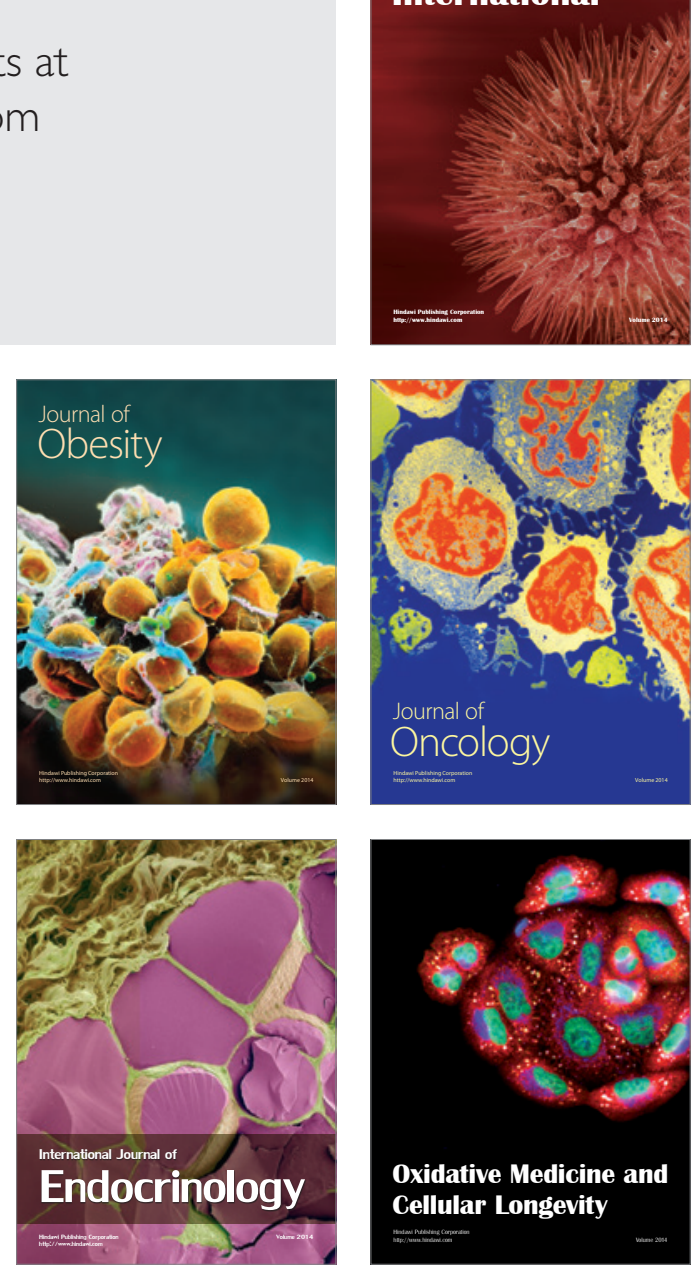